\title{
Ten-Eleven Translocation-2 gene mutations: A potential new molecular marker in malignant gliomas (Review)
}

\author{
LEI YU and SONGTAO QI
}

\author{
Department of Neurosurgery, Nanfang Hospital, Southern Medical University, Guangzhou 510515, P.R. China
}

Received May 26, 2011; Accepted October 10, 2011

DOI: $10.3892 / \mathrm{ol} .2011 .447$

\begin{abstract}
Alterations of the Ten-Eleven Translocation-2 (TET2) gene in myeloid malignancies and isocitrate dehydrogenase $(I D H)$ gene mutations in gliomas and myeloid malignancies have recently been identified using molecular, comparative genomic hybridization and single nucleotide polymorphism array techniques. The mutations of the TET2 gene have been shown to be mutually exclusive with $I D H 1 / 2$ mutations in acute myeloid leukemia (AML) and evidence has been found to provide a biochemical basis for the mutual exclusivity of IDHI/2 and TET2 gene mutations. Based on mounting evidence, we aimed to investigate whether TET2 mutations may be identified as novel mutations in malignant gliomas without $I D H 1 / 2$ mutations, and indicate their possible significance in gliomas.
\end{abstract}

\section{Contents}

1. Introduction

2. IDH $1 / 2$ mutations in gliomas and myeloid malignancies

3. TET2 mutations in gliomas and myeloid malignancies

4. Correlation between TET2 and IDH1/2 mutations

5. Discussion

\section{Introduction}

Malignant gliomas, the most common and lethal brain tumors, exhibit a great deal of diversity in their location, pathology, genetic status and response to therapy. Nevertheless, survival for patients with glioblastoma, the most aggressive glioma, although individually variable, has recently improved in the

Correspondence to: Professor SongTao Qi and Dr Lei Yu, Department of Neurosurgery, Nanfang Hospital, Southern Medical University, No. 1838 Guangzhou Avenue North, Guangzhou 510515, P.R. China

E-mail: yuleiguisi@gmail.com; ylgs2011@gmail.com

Key words: gene mutation, glioma, IDH1, IDH2, myeloid malignancies, TET2 last 5 years from an average of 10 to 14 months after diagnosis due to improvements in the standard of care (1). A comprehensive understanding of the genetic basis and pathology of gliomas has provided new information regarding biologically based tumor classification and has identified molecular prognostic biomarkers to improve the management of patients with gliomas. Over the past decades the application of sequencing, comparative genomic hybridization $(\mathrm{CGH})$ and single nucleotide polymorphism (SNP) array techniques have revealed the molecular genetic background of neoplasms through the identification of novel oncogenes and tumor suppressor genes.

\section{IDH1/2 mutations in gliomas and myeloid malignancies}

One recently identified class of genes mutated in cancer are those coding for isocitrate dehydrogenase (IDH), which catalyzes the oxidative decarboxylation of isocitrate to $\alpha$-ketoglutarate $(\alpha-K G)$ leading to NADPH production $(2,3)$. The $\mathrm{NADP}^{+}$-dependent IDH1 (located in the cytoplasm and peroxisomes) and its mitochondrial counterpart IDH2 are mutated in up to $75 \%$ of grade II-III gliomas and secondary glioblastoma multiforme (GBM) as well as myeloid malignancies including primary and secondary acute myeloid leukemia (AML, 15-30\%) and preleukemic clonal malignancies, such as myelodysplasia and myeloproliferative neoplasms (2-9). IDH1 mutation has rapidly emerged as a novel prognostic and diagnostic marker with which to identify low-grade gliomas and to distinguish secondary from primary GBM $(4,10,11)$. IDH1/2 mutations are heterozygous, with tumors retaining one wild-type copy of the relevant $I D H 1$ or $I D H 2$ allele and producing single amino acid substitutions at arginine 132 (R132) in IDH1 or corresponding arginine 172 (R172) in IDH2 in glioma and leukemia, or at arginine 140 (R140) in IDH2 in leukemia $(8,12)$. Notably, the R132 mutation in IDH1 results not only in a marked decrease of normal catalytic activity, but also in a gain of novel function catalyzing the $\mathrm{NADPH}^{+}$-dependent reduction of $\alpha$-KG to $\mathrm{R}(-)-2$-hydroxyglutarate $(2-\mathrm{HG})(8,12)$. Decreased $\alpha-K G$ and $\alpha-K G$-dependent prolyl hydroxylase (PHD) activity leads to an increase in hypoxia-inducible factor- $1 \alpha$ (HIF-1 $\alpha)$, since PHDs use $\alpha-\mathrm{KG}$ as a substrate for a reaction that normally targets HIF-1 $\alpha$ for degradation (13). $\alpha-K G$ reduction and $\mathrm{D}-2-\mathrm{HG}$ accumulation cooperatively contribute to tumorigenesis (14), which may resolve the continuing controversy over whether mutant $I D H 1$ is a tumor suppressor gene or an oncogene $(15,16)$. 


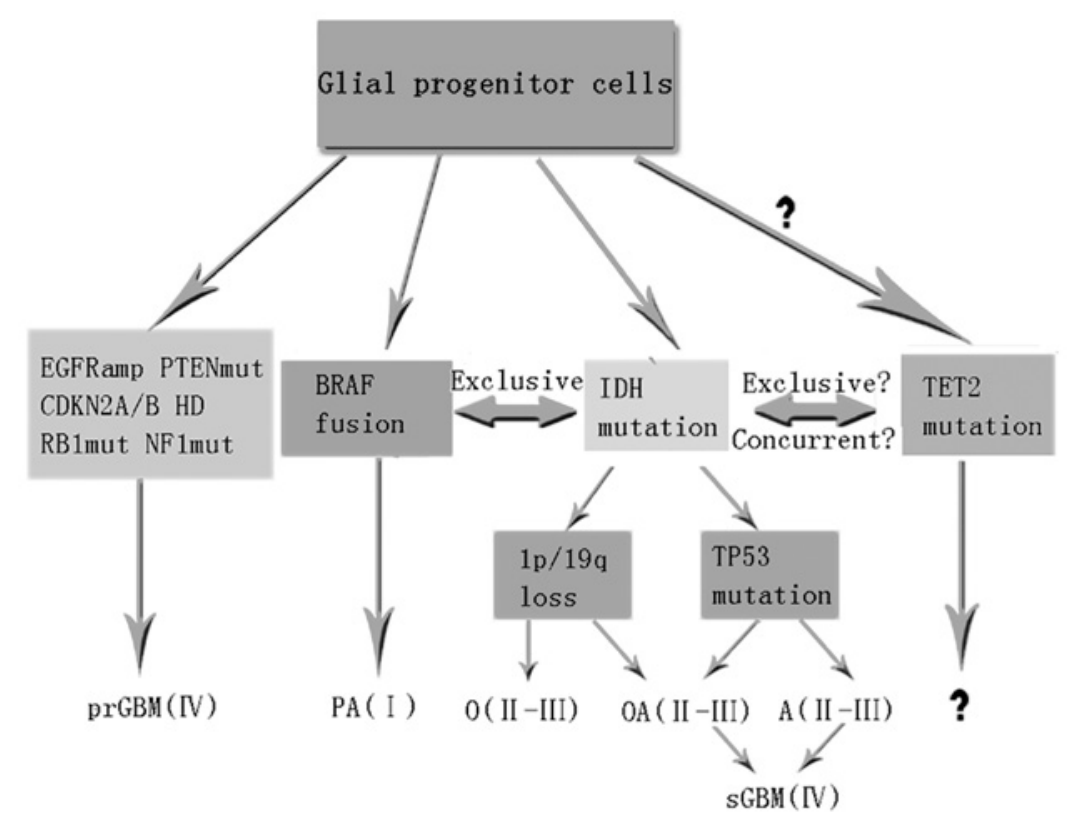

Figure 1. A model for tumorigenesis and progression of gliomas based on genetic alterations. TET2 mutations are integrated into the model, but their role in gliomas is unknown. PAI, pilocytic astrocytomas; OII, oligodendrogliomas; OAII, oligoastrocytomas; AII, diffuse astrocytomas; OIII, anaplastic oligodendrogliomas; OAIII, anaplastic oligoastrocytomas; AIII, anaplastic astrocytomas; sGBMIV, secondary glioblastomas; prGBM IV, primary glioblastomas occurring de novo; EGFR, epidermal growth factor receptor; PTEN, phosphatase and tensin homolog; CDKN2A and 2B , cyclin-dependent kinase inhibitor 2A and 2B; HD, homozygous deletion; RB1, retinoblastoma 1; mut, mutation; NF1, neurofibromatosis type 1; BRAF fusion, v-raf murine sarcoma viral oncogene homolog B1; IDH, isocitrate dehydrogenase; TP53, tumor protein p53; 1p/19q loss, homozygous deletion of chromosome arms 1p and 19q; TET2, Ten-Eleven Translocation-2 gene.

\section{TET2 mutations in gliomas and myeloid malignancies}

Other recently reported gene mutations are those of the Ten-Eleven Translocation-2 gene (TET2), which have been found in 20-26\% of myelodysplastic syndromes or secondary AML $(17,18), 14 \%$ of myeloproliferative neoplasms $(17,19,20)$, and $12 \%$ of de novo AML (21). TET2 is a putative tumor suppressor gene located at chromosome $4 \mathrm{q} 24$, encoding a dioxygenase that converts 5-methylcytosine to 5-hydroxymethylcytosine, leading to DNA demethylation at selective loci. It has also been indicated in the regulation of normal myelopoiesis (22-24), and the disruption of TET2 enzymatic activity favours myeloid tumorigenesis (24). Measurement of 5-hydroxymethylcytosine levels in myeloid malignancies may prove valuable as a diagnostic and prognostic tool to tailor therapies and assess responses to anticancer drugs (24). TET2 abnormalities are highly heterogeneous and inactivating showing a relatively diverse pattern of frame shift, nonsense, and missense mutations scattered across several of its exons. Additionally, TET2 may occur as hemizygous or heterozygous alterations, including loss of heterozygosity, due to hemizygous deletion or uniparental disomy $(17,25)$. In contrast to the affirmatively prognostic value of IDHI mutation in gliomas $(4,26)$, the prognostic value of TET2 mutations in myeloid malignancies remains unclear (27). Similarly, survival in primary AML did not appear to be affected by the presence of IDH mutations $(2,8,28)$.

\section{Correlation between $T E T 2$ and IDH1/2 mutations}

Mutations of TET2 gene have been shown to coexist with other pathogenetically relevant mutations, including the retinoic acid receptor $\alpha$ gene, thrombopoietin receptor gene (myeloprolifer- ative leukemia, MPL), janus kinase 2 gene (JAK2), KIT gene, FMS-like tyrosine kinase 3 gene (FLT3), renin-angiotensin system (RAS) gene, mixed lineage leukemia (MLL) gene, CCAAT enhancer binding protein $\alpha$ gene (CEBA+) or nucleophosmin 1 gene (NHMI) $(18-21,29,30)$, but occur in a manner mutually exclusive with that of $I D H 1$ and $I D H 2$ genes in AML (30). Xu et al found that the expression of mutant IDH1/2 and D-2-HG inhibited the activity of TET2 in catalyzing the $5 \mathrm{mC}$-to-5hmC conversion, which not only supported, but also provided a biochemical basis for the mutual exclusivity of IDH1/2 and TET2 gene mutations (14). The mechanisms of various gene mutations involved in disease initiation and/or progression are not isolated but associated, compatible or exclusive of each other. Considering the ubiquitous nature of mutant TET2 in myeloid malignancies, mutant $I D H 1 / 2$ in gliomas and both mutations in myeloid malignancies, it may be possible that TET2 mutation is also present in malignant gliomas, particularly those without $I D H 1 / 2$ mutation.

\section{Discussion}

Mounting evidence has indicated that TET2 alterations are a common event in a spectrum of myeloid malignancies, that IDH1/2 mutations are frequently present in gliomas, and that they both exhibit similar frequencies in myeloid malignancies. IDH 1/2 mutations were mutually exclusive with mutations in the $\alpha$-KG-dependent enzyme TET2, and TET2 loss-of-function mutations were associated with similar epigenetic defects to those of IDH1/2 mutants in AML. Expression of mutant IDH1/2 or TET2 depletion impaired hematopoietic differentiation and increased stem/progenitor cell marker expression, indicating a shared proleukemogenic effect (30). Additionally, 
the two mutations of IDHI and TET2 have been reported to occur at a relatively early stage during glioma and leukemia development $(17,18,31)$. These findings suggest that TET2 mutations may be identified as novel mutations in malignant gliomas without $I D H 1 / 2$ mutations, since no reports are currently available regarding the association between TET2 mutations and malignant gliomas.

IDH1/2 mutations are concurrent with TP53 mutations in astrocytic tumors $(9,31,32)$ and $1 \mathrm{p} 19 \mathrm{q}$ codeletion in oligodendroglial tumors $(9,32,33)$, but mutually exclusive with epidermal grow th factor receptor (EGFR) amplification (34) and the BRAF fusion gene (10,35). IDH1/2 mutations are inversely associated with numerous characteristic genetic changes of primary glioblastomas, including EGFR amplification, cyclin-dependent kinase inhibitor $2 \mathrm{~A}$ or $2 \mathrm{~B}$ deletion, and phosphatase and tensin homolog mutations $(9,32)$. IDH mutations do not increase in frequency in the progression to higher-grade gliomas and occur prior to other genetic changes, indicating that these mutations arise at some point in the transition from glial progenitor cells to a clinically evident tumor $(9,31,32,36,37)$. Common genetic changes associated with various glioma subtypes are shown in Fig. 1 in addition to the hypothesis that TET2 mutations occur in gliomas. If the above-mentioned hypothesis holds true, several questions may be raised. The specific type of TET2 mutation in malignant gliomas remains to be identified. It is also unknown whether TET2 mutations are exclusive with $I D H 1 / 2$ mutations in gliomas, or perhaps even concurrent with them. In instances where mutant TET 2 coexists with IDH1/2 mutations or other mutations, it is currently unclear as to whether it predates or postdates their emergence. Within the context of a specific disease, it is likely that the presence of mutant TET2 affects phenotype, prognosis or treatment response. Finally, it is necessary to determine the pathogenetic contribution of mutant TET2 in cancer, particularly in view of its occurrence across different molecular profiles.

Further clinical investigation from different centers is required to confirm our hypothesis. Additional laboratory studies are required to clarify the biological consequence of these mutations prior to any clinical applications.

\section{References}

1. Van Meir EG, Hadjipanayis CG, Norden AD, Shu HK, Wen PY and Olson JJ: Exciting new advances in neuro-oncology: the avenue to a cure for malignant glioma. CA Cancer J Clin 60: 166-193, 2010.

2. Mardis ER, Ding L, Dooling DJ, et al: Recurring mutations found by sequencing an acute myeloid leukemia genome. $\mathrm{N}$ Engl J Med 361: 1058-1066, 2009.

3. Parsons DW, Jones S, Zhang X, et al: An integrated genomic analysis of human glioblastoma multiforme. Science 321: $1807-1812,2008$.

4. Kloosterhof NK, Bralten LB, Dubbink HJ, French PJ and van den Bent MJ: Isocitrate dehydrogenase-1 mutations: a fundamentally new understanding of diffuse glioma? Lancet Oncol 12: 83-91, 2011.

5. Marcucci G, Maharry K, Wu YZ, et al: IDH1 and IDH2 gene mutations identify novel molecular subsets within de novo cytogenetically normal acute myeloid leukemia: a Cancer and Leukemia Group B study. J Clin Oncol 28: 2348-2355, 2010.

6. Paschka P, Schlenk RF, Gaidzik VI, et al: IDH1 and IDH2 mutations are frequent genetic alterations in acute myeloid leukemia and confer adverse prognosis in cytogenetically normal acute myeloid leukemia with NPM1 mutation without FLT3 internal tandem duplication. J Clin Oncol 28: 3636-3643, 2010 .
7. Tefferi A, Lasho TL, Abdel-Wahab O, et al: IDH1 and IDH2 mutation studies in 1473 patients with chronic-, fibrotic- or blast-phase essential thrombocythemia, polycythemia vera or myelofibrosis. Leukemia 24: 1302-1309, 2010.

8. Ward PS, Patel J, Wise DR, et al: The common feature of leukemia-associated IDH1 and IDH2 mutations is a neomorphic enzyme activity converting alpha-ketoglutarate to 2-hydroxyglutarate. Cancer Cell 17: 225-234, 2010.

9. Yan H, Parsons DW, Jin G, et al: IDH1 and IDH2 mutations in gliomas. N Engl J Med 360: 765-773, 2009.

10. Ducray F, El-Hallani S and Idbaih A: Diagnostic and prognostic markers in gliomas. Curr Opin Oncol 21: 537-542, 2009.

11. Schiff D and Purow BW: Neuro-oncology: isocitrate dehydrogenase mutations in low-grade gliomas. Nat Rev Neurol 5: 303-304, 2009.

12. Dang L, White DW, Gross S, et al: Cancer-associated IDH1 mutations produce 2-hydroxyglutarate. Nature 462: 739-744, 2009.

13. Zhao S, Lin $\mathrm{Y}, \mathrm{Xu} \mathrm{W}$, et al: Glioma-derived mutations in IDH1 dominantly inhibit IDH1 catalytic activity and induce HIF-1alpha. Science 324: 261-265, 2009.

14. Xu W, Yang H, Liu Y, et al: Oncometabolite 2-hydroxyglutarate is a competitive inhibitor of alpha-ketoglutarate-dependent dioxygenases. Cancer Cell 19: 17-30, 2011.

15. Garber K: Oncometabolite? IDH1 discoveries raise possibility of new metabolism targets in brain cancers and leukemia. J Natl Cancer Inst 102: 926-928, 2010.

16. Zhu J, Zuo J, Xu Q, Wang X, Wang Z and Zhou D: Isocitrate dehydrogenase mutations may be a protective mechanism in glioma patients. Med Hypotheses 76: 602-603, 2011.

17. Delhommeau F, Dupont S, Della VV, et al: Mutation in TET2 in myeloid cancers. N Engl J Med 360: 2289-2301, 2009.

18. Langemeijer SM, Kuiper RP, Berends M, et al: Acquired mutations in TET2 are common in myelodysplastic syndromes. Nat Genet 41: 838-842, 2009.

19. Tefferi A, Levine RL, Lim KH, et al: Frequent TET2 mutations in systemic mastocytosis: clinical, KITD816V and FIP1L1-PDGFRA correlates. Leukemia 23: 900-904, 2009.

20. Tefferi A, Pardanani A, Lim KH, et al: TET2 mutations and their clinical correlates in polycythemia vera, essential thrombocythemia and myelofibrosis. Leukemia 23: 905-911, 2009.

21. Abdel-Wahab O, Mullally A, Hedvat C, et al: Genetic characterization of TET1, TET2, and TET3 alterations in myeloid malignancies. Blood 114: 144-147, 2009.

22. Ito S, D'Alessio AC, Taranova OV, Hong $\mathrm{K}$, Sowers LC and Zhang Y: Role of Tet proteins in $5 \mathrm{mC}$ to $5 \mathrm{hmC}$ conversion, ES-cell self-renewal and inner cell mass specification. Nature 466: 1129-1133, 2010.

23. Tahiliani M, Koh KP, Shen Y, et al: Conversion of 5-methylcytosine to 5-hydroxymethylcy tosine in mammalian DNA by MLL partner TET1. Science 324: 930-905, 2009.

24. Ko M, Huang Y, Jankowska AM, et al: Impaired hydroxylation of 5-methylcytosine in myeloid cancers with mutant TET2. Nature 468: 839-843, 2010.

25. Jankowska AM, Szpurka H, Tiu RV, et al: Loss of heterozygosity 4q24 and TET2 mutations associated with myelodysplastic/ myeloproliferative neoplasms. Blood 113: 6403-6410, 2009.

26. Reitman ZJ and Yan H: Isocitrate dehydrogenase 1 and 2 mutations in cancer: alterations at a crossroads of cellular metabolism. J Natl Cancer Inst 102: 932-941, 2010.

27. Tefferi A: Novel mutations and their functional and clinical relevance in myeloproliferative neoplasms: JAK2, MPL, TET2, ASXL1, CBL, IDH and IKZF1. Leukemia 24: 1128-1138, 2010.

28. Chou WC, Hou HA, Chen CY, et al: Distinct clinical and biologic characteristics in adult acute myeloid leukemia bearing the isocitrate dehydrogenase 1 mutation. Blood 115: 2749-2754, 2010.

29. Tefferi A, Lim KH, Abdel-Wahab O, et al: Detection of mutant TET2 in myeloid malignancies other than myeloproliferative neoplasms: CMML, MDS, MDS/MPN and AML. Leukemia 23: 1343-1345, 2009.

30. Figueroa ME, Abdel-Wahab O, Lu C, et al: Leukemic IDH1 and IDH2 mutations result in a hypermethylation phenotype, disrupt TET2 function, and impair hematopoietic differentiation. Cancer Cell 18: 553-567, 2010.

31. Watanabe T, Nobusawa S, Kleihues P and Ohgaki H: IDH1 mutations are early events in the development of astrocytomas and oligodendrogliomas. Am J Pathol 174: 1149-1153, 2009.

32. Ichimura K, Pearson DM, Kocialkowski S, et al: IDH1 mutations are present in the majority of common adult gliomas but rare in primary glioblastomas. Neuro Oncol 11: 341-347, 2009. 
33. Labussiere M, Idbaih A, Wang XW, et al: All the 1p19q codeleted gliomas are mutated on IDH1 or IDH2. Neurology 74: 1886-1890, 2010.

34. Sanson M, Marie Y, Paris S, et al: Isocitrate dehydrogenase 1 codon 132 mutation is an important prognostic biomarker in gliomas. J Clin Oncol 27: 4150-4154, 2009.

35. Korshunov A, Meyer J, Capper D, et al: Combined molecular analysis of BRAF and IDH1 distinguishes pilocytic astrocytoma from diffuse astrocytoma. Acta Neuropathol 118: 401-405, 2009.
36. Balss J, Meyer J, Mueller W, Korshunov A, Hartmann C and von Deimling A: Analysis of the IDH1 codon 132 mutation in brain tumors. Acta Neuropathol 116: 597-602, 2008.

37. Ohgaki $\mathrm{H}$ and Kleihues P: Genetic alterations and signaling pathways in the evolution of gliomas. Cancer Sci 100: 2235-2241, 2009. 